\begin{abstract}
A MODEL OF
THE MAGNETOSPHERIC TEMPERATURE DISTRIBUTION

by

H. G. Mayr and H. Volland

Aeronomy Branch
\end{abstract}

GODDARD SPACE FLIGHT CENTER

Greenbelt, Maryland 


\begin{abstract}
In the magnetosphere, the equation of heat flux parallel to the magnetic field must be modified because of the low electron density and large mean free path. This leads to a density-dependent heat conductivity which is decreased such that the high temperatures in the magnetosphere observed by Serbu and Maier can be explained. In the upper magnetosphere, turbulent heat transfer in accordance with Bohm's diffusion coefficient can account for heat fluxes on the order of $10^{-10} \mathrm{erg} / \mathrm{cm}^{2} \mathrm{sec}$ across the magnetic field. Such heat fluxes are consistent with ion-electron temperature differences of $\mathrm{T}_{\mathrm{i}}-\mathrm{T}_{\mathrm{e}} \sim 10^{4}{ }^{\circ} \mathrm{K}$ also discovered by Serbu and Maier. This direct thermal energy input is negligible for the energy balance of the magnetosphere when compared with the heat fluxes of $10^{-3}-10^{-2} \mathrm{erg} / \mathrm{cm}^{2} \mathrm{sec}$ generated through non-local heating by fast ionospheric electrons.
\end{abstract}




\section{CONTENTS}

Abstract $\ldots \ldots \ldots \ldots \ldots \ldots \ldots \ldots \ldots \ldots \ldots \ldots \ldots \ldots \ldots \ldots \ldots \ldots \ldots$

INTRODUCTION $\ldots \ldots \ldots \ldots \ldots \ldots \ldots \ldots \ldots \ldots \ldots \ldots \ldots \ldots \ldots$

PARALLEL HEAT CONDUCTION .................. 4

TURBULENT PERPENDICULAR HEAT TRANSFER .......... 12

CONCLUSIONS $\ldots \ldots \ldots \ldots \ldots \ldots \ldots \ldots \ldots \ldots \ldots \ldots \ldots \ldots \ldots \ldots$

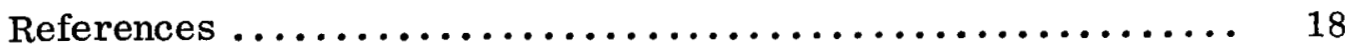




\title{
A MODEL OF
}

THE MAGNETOSPHERIC TEMPERATURE DISTRIBUTION

\author{
by \\ H. G. Mayr* and H. Volland ${ }^{\dagger}$ \\ Goddard Space Flight Center
}

\section{INTRODUCTION}

Measurements of the low-energy electron spectrum by Serbu and Maier [1966] on IMP II have shown that in the magnetosphere these electrons have an almost Maxwellian velocity distribution.

In the equatorial plane the electron temperature $\mathrm{T}_{\mathbf{e}}$ increases with distance $r$ from the earth's center as

$$
\mathrm{T}_{\mathrm{e}} \propto \mathrm{r}^{2}
$$

reaching values of the order $\mathrm{T}_{\mathrm{e}} \sim 20,000^{\circ} \mathrm{K}$ at heights between $5 \mathrm{a}$ and $15 \mathrm{a}$ where $a$ is the earth radius. Otherwise relatively low temperatures of the order of $3000^{\circ} \mathrm{K}$ have been observed at the base of the protonosphere at heights of about $1000 \mathrm{~km}$ above the ground at low and high latitudes [Brace, Reddy and Mayr, 1967].

According to the classical formulas of heat conduction within an anisotropic plasma, the predominant heat flow parallel to the geomagnetic field within the protonosphere is due to electrons [see, e.g., Kaufman, 1966]. The coefficient of heat conductivity of electrons is

$$
\kappa_{11} \sim \kappa^{(e)}=\mathrm{A}_{1} \mathrm{~T}^{5 / 2} \mathrm{erg} / \mathrm{cm} \mathrm{sec} \mathrm{deg},
$$

\footnotetext{
* National Academy of Sciences - National Research Council Associate with the National Aeronautics and Space Administration.

ton leave from the Astronomical Institutes of the University of Bonn, Germany, as a National Academy of Sciences - National Research Council Associate with the National Aeronautics and Space Administration.
} 
with

$$
A_{1} \sim 1 \times 10^{-6}
$$

Perpendicular to the magnetic field the ionic component predominates and its coefficient of heat conductivity is

$$
\kappa_{\perp} \sim \kappa_{\perp}{ }^{(i)}=\kappa^{(\mathrm{e})} \frac{\nu_{\mathrm{i}}{ }^{2}}{\omega_{\mathrm{i}}{ }^{2}} \sqrt{\frac{\mathrm{m}_{\mathrm{e}}}{\mathrm{m}_{\mathrm{i}}}}=\mathrm{A}_{2} \frac{\mathrm{N}_{\mathrm{i}}^{2}}{\mathrm{~B}^{2} \mathrm{~T}_{\mathrm{i}}^{1 / 2}} \text { erg } / \mathrm{cm} \mathrm{sec} \mathrm{deg,} \mathrm{(3)}
$$

with

$$
\mathrm{A}_{2} \sim 3 \times 10^{-16} .
$$

Here $\mathrm{T}_{\mathbf{i}}, \mathrm{N}_{\mathbf{i}}$, and $\nu_{\mathbf{i}}$ are temperature, density, and collision number of the protons; $B$ is the earth's magnetic field, $\omega_{i}$ the ion gyrofrequency, $m_{i}$ the ion mass, and $m_{e}$ is the electron mass.

Taking mean numerical values of $\mathrm{T}_{\mathrm{e}} \sim \mathrm{T}_{\mathrm{i}} \sim 10^{4}{ }^{\circ} \mathrm{K}, \mathrm{N}_{\mathrm{i}} \sim 10^{2} \mathrm{~cm}^{-3}$ and $\mathrm{B} \sim 10^{-3}$ gauss, leads to a ratio between the coefficients of heat conductivity of

$$
\frac{\kappa_{\perp}}{\kappa_{11}}=3 \times 10^{-12}
$$

thus, one must expect an extremely large anisotropy of temperature: namely, near-isothermy parallel to the field lines and almost complete heat-insulation perpendicular to the field lines.

To derive a first approximation of the temperature profile within the protonosphere, we consider a heat-insulated field line and neglect the geometrics of the dipole field. The static energy equation has then the form

$$
\operatorname{div} \vec{j}=-\operatorname{div}(\kappa \operatorname{grad} T)=Q \text {, }
$$

where $\vec{j}$ is the heat flux vector and $Q$ is the external energy input per unit volume. Introducing the coefficient $\kappa_{11}$ of (2) into (5) and taking $Q=0$ leads to the solution 


$$
\mathrm{T}=\left[\mathrm{T}_{\mathrm{I}}{ }^{7 / 2}+\frac{7}{2} \frac{\kappa_{\mathrm{I}} \mathrm{T}_{\mathrm{I}}^{\prime}}{\mathrm{A}_{\mathrm{i}}}\left(\mathrm{s}-\mathrm{s}_{\mathrm{I}}\right)\right]^{-2 / 7} \sim \mathrm{C} \mathrm{s}^{2 / 7}
$$

where $T_{I}$ and $T_{I}^{\prime}=\left(\frac{\partial T}{\partial s}\right)_{I}$ are the lower boundary values at height $s_{I^{\bullet}}$. From Equation 6 follows the expected small temperature increase (as $\mathrm{s}^{2 / 7}$ ) parallel to the field at greater heights where $\left(s \gg s_{I}\right)$. To maintain even this small gradient and to reach a temperature $\mathrm{T}_{\mathrm{I}}=20,000^{\circ} \mathrm{K}$ at the top of the field line, a heat flux of the order of

$$
\mathrm{j}_{\mathrm{I}}=\kappa_{\mathrm{I}} \mathrm{T}_{\mathrm{I}}^{\prime}=1 \mathrm{erg} / \mathrm{cm}^{2} \mathrm{sec}
$$

had to flow into the thermosphere at medium and higher latitudes. This would imply a temperature gradient of the order

$$
\mathrm{T}_{\mathrm{I}}^{\prime}=100^{\circ} / \mathrm{km}
$$

at a height of $1000 \mathrm{~km}$. Tamao [1966], taking into account the geometry of the field lines, arrived at a similar result. He assumed that this heat flow must be supplied from interplanetary space via turbulent heat transfer across the field lines at greater heights. But the observations made by Brace, Reddy and Mayr [1967] suggest an upper limit of the electron temperature gradient at $1000 \mathrm{~km}$ that is smaller by at least two orders of magnitude:

$$
\mathrm{T}_{\mathrm{I}}^{\prime} \sim 1^{\circ} / \mathrm{km} \text {. }
$$

If we introduce this value into (6) together with $\mathrm{T}_{\mathrm{I}}=3000^{\circ} \mathrm{K}$, we obtain a temperature at the top of the field lines of $\mathrm{T}_{\mathrm{II}} \sim 7000^{\circ} \mathrm{K}$ at a distance $r=8 \mathrm{a}$. This temperature is considerably smaller than that obtained by Serbu and Maier [1966].

The following investigation seeks to resolve this striking discrepancy. A more rigorous treatment of heat conduction shows that the heat conductivity is effectively decreased in regions of low electron densities, causing a general increase of temperature at greater heights.

Furthermore, the effect of turbulent heat transfer perpendicular to the magnetic field lines is estimated. This leads to an understanding of the 
ion-electron temperature difference observed by Serbu and Maier [1967] in the upper magnetosphere.

\section{PARALLEL HEAT CONDUCTION}

A characteristic quantity for heat conduction is the mean free path. This quantity is given for electrons by

$$
\lambda=\mathrm{K} \frac{\mathrm{T}_{\mathrm{e}}{ }^{2}}{\mathrm{~N}_{\mathrm{e}}} ; \mathrm{K} \sim 3 \times 10^{4}
$$

and is ordinarily related to the parallel heat conductivity through

$$
\kappa_{11}^{(\mathrm{e})} \propto \overline{\mathrm{c}} \lambda \mathrm{N}_{\mathrm{e}}
$$

where $\bar{c}$ is the mean thermal velocity $\left(\bar{c} \propto T_{e}{ }^{1 / 2}\right)$.

Equation (8) is valid only if the temperature variation can be considered linear within the range of the mean free path, and if the mean free path is almost constant over the same range; only then does (7) have real significance.

In the magnetosphere (where $\mathrm{N}$ can be on the order of $10^{1}-10^{2} \mathrm{~cm}^{-3}$ and $\mathrm{T}_{\mathbf{e}}$ can be on the order of $10^{4} \mathrm{~K}$ ) the mean free path (as defined in (7)) is on the order of $10^{4}-10^{6} \mathrm{~km}$. (This distance is comparable with or exceeds the dimensions of the plasma confined in field tubes.) In the light of Serbu and Maier's measurements it is obvious that the temperature variation is no longer linear over such a distance and that the mean free path is strongly variable. Thus, (8) is no longer valid, and we must modify the heat-conduction equation so as to take into account this new situation in the magnetosphere.

In this paper, we approach the problem by means of a modified meanfree-path method. This is not precisely a rigorous treatment; rather, it seeks 
to prove that heat conductivity becomes density-dependent and is marked by a decrease in the magnetosphere.

Expanding the temperature into a power series in $s$ around $s_{0}=0$ gives

$$
T=T_{0}+\left(\frac{\partial T}{\partial s}\right)_{0} s+\frac{1}{2}\left(\frac{\partial^{2} T}{\partial s^{2}}\right)_{0} s^{2}+\cdots \cdot
$$

where higher terms are assumed to be negligibly small. If $\zeta$ is the angle of the velocity direction toward the s axis, each electron carries the energy

$$
\begin{aligned}
& \frac{3}{2} \mathrm{kT}_{0}-\lambda_{-} \cos \zeta\left[\frac{\partial}{\partial \mathrm{s}}\left(\frac{3}{2} \mathrm{kT}\right)\right]_{0} \\
& +\frac{\lambda_{-}^{2}}{2} \cos ^{2} \zeta\left[\frac{\partial^{2}}{\partial \mathrm{s}^{2}}\left(\frac{3}{2} \mathrm{kT}\right)\right]_{0}+\cdots
\end{aligned}
$$

through the plane $s=0$. The quantity $\lambda_{-}$is considered the mean free path of the electrons in the region where $s<0$; $k$ is Boltzmann's constant. Correspondingly, electrons going in the opposite direction transport the energy

$$
\begin{aligned}
& \frac{3}{2} \mathrm{kT}_{0}+\lambda+\cos \zeta\left[\frac{\partial}{\partial \mathrm{s}}\left(\frac{3}{2} \mathrm{kT}\right)\right]_{0} \\
& +\frac{\lambda_{+}^{2}}{2} \cos ^{2} \zeta\left[\frac{\partial^{2}}{\partial \mathrm{s}^{2}}\left(\frac{3}{2} \mathrm{kT}\right)\right]_{0}+\cdots
\end{aligned}
$$

where $\lambda_{+}$is the mean free path in the region where $s>0$. The difference of these energies is 


$$
\begin{aligned}
& -\left(\lambda_{+}+\lambda_{-}\right) \cos \zeta\left[\frac{\partial}{\partial s}\left(\frac{3}{2} \mathrm{kT}\right)\right]_{0} \\
& -\frac{1}{2}\left(\lambda_{+}{ }^{2}-\lambda_{-}{ }^{2}\right) \cos ^{2} \zeta\left[\frac{\partial^{2}}{\partial s^{2}}\left(\frac{3}{2} \mathrm{kT}\right)\right]_{0}
\end{aligned}
$$

During unit time $\mathrm{c} \cos \zeta \mathrm{N}_{\mathrm{e}} \mathrm{fd} \zeta$ electrons traverse the unit area at $s=0$, if $f$ is the distribution function for the velocities $c$, given by

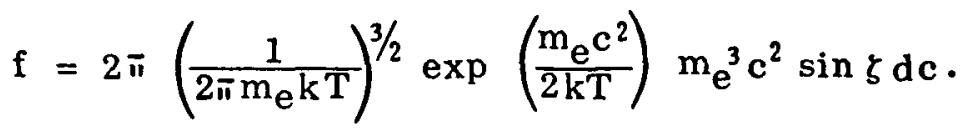

The net energy transported by electrons with velocity $\mathrm{c}$ and angle $\zeta$ is therefore

$$
\begin{aligned}
-\left(\lambda_{+}+\lambda_{-}\right) c \frac{\partial}{\partial s}\left(\frac{3}{2} k T\right) & N_{e} f \cos ^{2} \zeta d \zeta \\
& -\frac{1}{2}\left(\lambda_{+}^{2}-\lambda_{-}^{2}\right) c \frac{\partial^{2}}{\partial s^{2}}\left(\frac{3}{2} k T\right) N_{e} f \cos ^{3} \zeta d \zeta .
\end{aligned}
$$

Integration over $0 \leq \zeta \leq \frac{\pi}{2}$ and $0 \leq \mathrm{c} \leq \infty$ leads in a straightforward manner to heat conduction flux in the form

$$
j=-\frac{k}{4} \bar{c}\left(\lambda_{+}+\lambda_{-}\right) N_{e} \frac{\partial T}{\partial s}-\frac{3 k}{32} \bar{c}\left(\lambda_{+}{ }^{2}-\lambda_{-}{ }^{2}\right) N_{e} \frac{\partial^{2} T}{\partial s^{2}}
$$

If we assume that the mean free path is almost constant in space, then $\lambda_{+}=\lambda_{-}$, and (14) reduces to the classical formula

$$
j_{c}=-\frac{k \bar{c}}{2} \lambda N_{e} \frac{\partial T}{\partial s}=-A_{1} T^{5 / 2} \frac{\partial T}{\partial s}
$$

where $\lambda$ is defined by (7). This shows that the additional term in the expansion of the heat-flux formula (equation 14) arises from the variability of the mean free path. 
The definitions of $\lambda_{+}$and $\lambda_{-}$are rather problematic. A reasonable definition of these quantities can be given in the form

$$
\int_{0}^{\lambda+} \frac{d s}{\lambda}=\int_{-\lambda_{-}}^{0} \frac{d s}{\lambda}=1
$$

where $\lambda$ is defined by (7). This leads to

$$
\frac{1}{\mathrm{~K}} \int_{0}^{\lambda_{+}} \frac{\mathrm{N}}{\mathrm{T}^{2}} \mathrm{ds}=\frac{1}{\mathrm{~K}} \int_{0}^{\lambda_{+}}\left[\mathrm{T}_{0}+\left(\frac{\partial \mathrm{T}}{\partial \mathrm{s}}\right)_{0} \mathrm{~s}+\frac{1}{2}\left(\frac{\partial^{2} \mathrm{~T}}{\partial \mathrm{s}^{2}}\right)_{0} \mathrm{~s}^{2}\right]^{-1} \mathrm{ds}=1,
$$

and

$$
\frac{1}{K} \int_{-\lambda_{-}}^{0} \frac{N}{T^{2}} d s=\frac{1}{K} \int_{-\lambda_{-}}^{0} N\left[T_{0}-\left(\frac{\partial T}{\partial s}\right)_{0} s+\frac{1}{2}\left(\frac{\partial^{2} T}{\partial s^{2}}\right)_{0} s^{2}\right]^{-1} d s=1
$$

A treatment of the mean free paths in this form would lead to the functions

$$
\lambda_{+}=\lambda_{+}\left(T, \frac{\partial T}{\partial s}, \frac{\partial^{i} T}{\partial s^{2}}\right)
$$

and

$$
\lambda_{-}=\lambda_{-}\left(T, \frac{\partial T}{\partial s}, \frac{\partial^{2} T}{\partial s^{2}}\right)
$$

Introducing the heat flux, (14), into the energy continuity, (5), and integrating up to the top of the field line (equator), leads to the differential equation

$$
\begin{aligned}
& \frac{k}{4} \bar{c}\left(\lambda_{+}+\lambda_{-}\right) N_{e} \frac{\partial T}{\partial s}+\frac{3 k}{32} \bar{c}\left(\lambda_{+}{ }^{2}-\lambda_{-}{ }^{2}\right) N_{e} \frac{\partial^{2} T}{\partial s^{2}}= \\
& =\frac{k}{4} \bar{c}_{\Pi}\left(\lambda_{+}+\lambda_{-}\right)_{\Pi} N_{e_{I I}}\left(\frac{\partial T}{\partial s}\right)_{I I}+\frac{3 k}{32} \bar{c}_{\Pi}\left(\lambda_{+}{ }^{2}-\lambda_{-}{ }^{2}\right)_{I I} N_{e_{\Pi}}\left(\frac{\partial^{2} T}{\partial s^{2}}\right)_{\Pi}+\int_{\mathbf{s}}^{s} \mathrm{Qds}^{s}
\end{aligned}
$$


Because of symmetry with respect to the equatorial plane, at $s=s_{J I}$ at the equator the conditions

$$
\left(\frac{\partial T}{\partial s}\right)_{\Pi}=0
$$

and

$$
\lambda_{+\Pi}=\lambda_{-\Pi}
$$

must be fulfilled. This yields the equation

$$
\frac{\mathrm{k}}{4} \overline{\mathbf{c}}\left(\lambda_{+}+\lambda_{-}\right) \mathrm{N}_{\mathrm{e}} \frac{\partial \mathrm{T}}{\partial \mathrm{s}}+\frac{3 \mathrm{k}}{32} \overline{\mathbf{c}}\left(\lambda_{+}{ }^{2}-\lambda_{-}{ }^{2}\right) \mathrm{N}_{\mathrm{e}} \frac{\partial^{2} \mathrm{~T}}{\partial \mathrm{s}^{2}}=\int_{\mathrm{s}}^{\mathrm{s} \Pi} \mathrm{Qds}
$$

for which a solution can be obtained if the temperature is known at some location $s_{I}\left(T=T_{I}\right)$.

Thus the problem is uniquely defined. The expansion of the heat flux in a form, (14), though it involves also second temperature derivatives, does not require an additional boundary condition for the solution of the energy equation.

A solution as outlined here is difficult, because of the complicated functions $\lambda_{+}$and $\lambda_{-}$(equations 16a and 17a). Therefore, we shall discuss a very specific magnetospheric condition that allows a rather simple approach.

In the heat-flux formula,(14), the second term, proportional to the second temperature derivative, is negative. This is true because the magnetospheric temperature distribution exhibits a maximum at the equator; and because $\lambda_{+}$is larger than $\lambda_{-}$for the observed positive temperature gradients in the magnetosphere. That term in (14) therefore decreases the heat flux and thus decreases what might be called the "effective conductivity." For a constant temperature at the base of the magnetosphere, this decrease of the heat conductivity leads to an increase of the electron temperature in the upper magnetosphere. For 
the sake of simplicity, we shall disregard this second term in (14) in our subsequent discussion; therefore, the derived temperature should be regarded as a lower limit.

From (16) and (17) it is evident that at the bottom of the magnetosphere, in a region where $\mathrm{N} \sim 10^{2} \mathrm{~cm}^{-3}$ and $\mathrm{T} \sim 4 \times 10^{3}{ }^{\circ} \mathrm{K}$

$$
\begin{aligned}
& \lambda_{+}>5 \times 10^{4} \mathrm{~km}, \\
& \lambda_{-}<5 \times 10^{4} \mathrm{~km},
\end{aligned}
$$

and consequently

$$
\left(\lambda_{+}+\lambda_{-}\right)>5 \times 10^{4} \mathrm{~km}
$$

Distances of this order are comparable with the length of field lines which, for $L=6$, are about $5 \times 10^{4} \mathrm{~km}$ from the earth surface up to the equatorial plane. Because of this, the upper limit of $\left(\lambda_{+}+\lambda_{-}\right)$cannot exceed the total length of the field line-which is $10^{5} \mathrm{~km}$; it follows that

$$
10^{5}>\left(\lambda_{+}+\lambda_{-}\right)>5 \times 10^{4} \mathrm{~km}
$$

As can easily be verified, this condition is also valid along the entire field line above the base of the magnetosphere.

Thus, it seems that under magnetospheric conditions of low densities the quantity $\lambda_{+}+\lambda_{-}$is almost constant. Its lower limit is close to the dimensions of the field tubes and its upper limit cannot exceed this. The heat flux in the magnetosphere then takes the form

$$
\mathrm{j} \sim-\frac{k \bar{c}}{4} \ell N_{e} \frac{\partial T}{\partial s} \sim-M \ell N_{e} T^{1 / 2} \frac{\partial T}{\partial s}
$$

where, after substitution for $\bar{c}, M$ is a constant and $\ell$ is the length of the field line.

A comparison of this modified heat-flux formula with the classical expression (equation 15) shows marked differences. The classical formula gives 
the conductivity as independent of density and increasing strongly with increasing temperatures. Equation 22 states that under magnetospheric conditions the heat conductivity becomes density-dependent (decreasing with density), and that the temperature dependence of the conductivity becomes very weak $\left(\propto \mathrm{T}^{1 / 2}\right)$ as compared with the classical conductivity $\left(\propto \mathrm{T}^{5 / 2}\right)$. Thus, in the magnetosphere the conductivity, because of its density-dependence, tends to decrease with height; in striking contrast to the behavior of the classical conductivity which, because of its temperature dependence, strongly tends to increase with height.

Combining the heat-flux equation 23 with the energy equation 5 leads to

$$
M \ell N T^{1 / 2} \frac{\partial T}{\partial s} \sim \int_{s}^{s} \mathrm{II} d s,
$$

which yields

$$
\mathrm{T}^{3 / 2}=\mathrm{T}_{\mathrm{I}}^{3 / 2}+\frac{3}{2} \frac{1}{\mathrm{Ml}} \int_{\mathrm{S}_{\mathrm{I}}}^{\mathrm{s}} \frac{\mathrm{ds}}{\mathrm{N}} \int_{\mathbf{S}}^{\mathrm{s}_{\mathrm{II}}} \mathrm{Qds} .
$$

If we assume that $\mathrm{T}_{\mathrm{I}}=4 \times 10^{3} \mathrm{~K}$ and $\mathrm{Q} \sim 3 \times 10^{-12}-$ a heating rate expected from fast electrons that escape the ionosphere [Geisler and Bowhill, 1965]-and that $\ell=10^{5}$, then we obtain a temperature distribution as shown in Figure 1 . This reveals a magnetospheric temperature structure that, at greater heights, is consistent with Serbu and Maier's measurements of temperatures on the order of $2 \times 10^{4} \mathrm{~K}$. Figure 1 is also consistent with the expected temperaturegradients at lower altitudes (on the order of $1^{\circ} \mathrm{K} / \mathrm{km}$, corresponding to energy fluxes on the order of $10^{-2} \mathrm{erg} / \mathrm{cm}^{2} \mathrm{sec}$ ).

We have shown that under magnetospheric conditions of low density the heat conductivity becomes dependent on density and almost independent of 


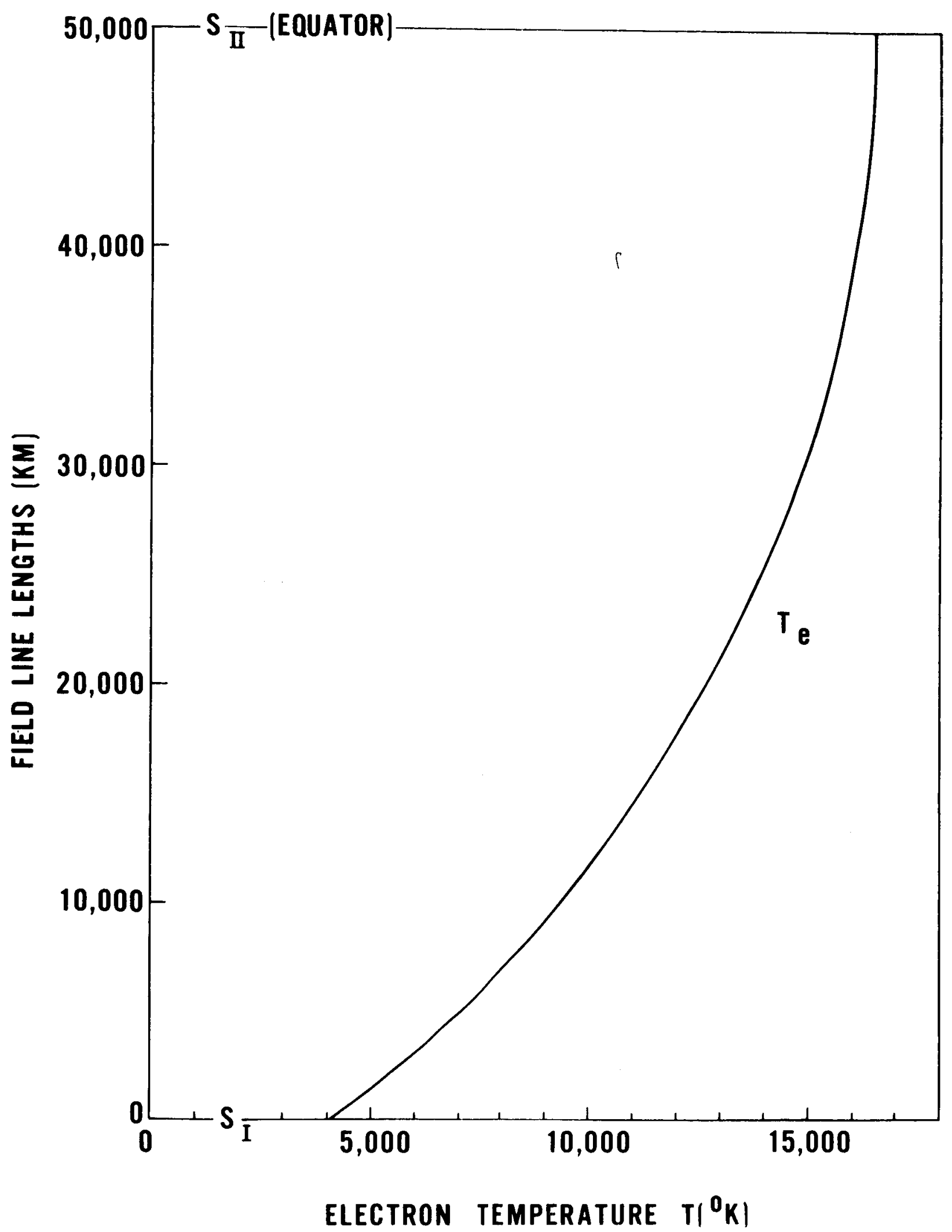

Figure 1. Calculated temperature distribution along a field line in the magnetosphere. 
temperature. As a consequence the heat conductivity is significantly decreased; and from this the observed unexpectedly high temperatures in the magnetosphere can be verified. The increase in equatorial temperature could thus be explained by the pronounced decrease of electron density observed by Carpenter and

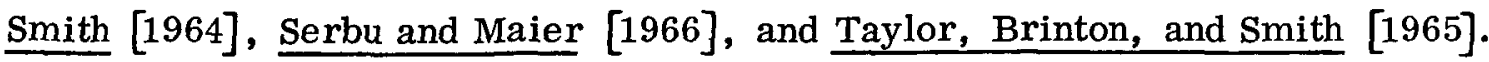

\section{TURBULENT PERPENDICULAR HEAT TRANSFER}

D. Bohm [1949] found, first from experiment and then from theory, that the effective perpendicular diffusion coefficient is enormously enhanced in a turbulent plasma. This coefficient $D_{B}$ is independent of density and related to temperature $\mathrm{T}$ and magnetic field $\mathrm{B}$ by the formula

$$
\mathrm{D}_{\mathrm{B}} \sim 5.4 \times 10^{2} \mathrm{~T} / \mathrm{B}[\underline{\text { Spitzer }} 1956]
$$

The coefficient of turbulent heat transfer is proportional to $D_{B}$ :

$$
\kappa_{\mathrm{B}}=\mathrm{kND}_{\mathrm{B}}
$$

At magnetospheric heights of 10 earth radii, this value is almost $10^{7}$ as large as the classical coefficient of heat transport based on collisions (equation 3). Combining (3), (24), and (25) (and equating $\mathrm{T}_{\mathrm{i}}$ to $\mathrm{T}$ and $\mathrm{N}_{\mathrm{i}}$ to $\mathrm{N}$ ), gives

$$
\frac{\kappa_{\mathrm{B}}}{\kappa_{\perp}} \propto \frac{\mathrm{B} \mathrm{T}^{3} / 2}{\mathrm{~N}}
$$

Assuming $\mathrm{B} \propto \mathrm{r}^{-3}$ and Serbu and Maier's [1966] experimentally derived temperature and density variations,

$$
\mathrm{T} \propto \mathbf{r}^{2}, \mathrm{~N} \propto \mathrm{r}^{-3}
$$


gives

$$
\frac{\kappa_{\mathrm{B}}}{\kappa_{\perp}} \propto \mathrm{r}^{3} .
$$

As we go farther from the earth, the heat-transfer coefficient based on Bohm's diffusion coefficient increases strongly as compared with the classical collision-type heat-transfer coefficient. Substituting in (25) the density and temperature distributions from (27) yields

$$
{ }^{\kappa}{ }_{\mathrm{B}} \sim 1.3 \times 10^{-6} \mathrm{r}^{2} / \mathrm{r}_{\mathrm{e}}^{2} \propto \mathrm{r}^{2}
$$

From (29) it can be shown that for a constant flux, the temperature gradient decreases as

$$
\frac{\partial T}{\partial r}=\frac{c}{r^{2} \kappa_{B}} \propto r^{-4}
$$

and may thus be responsible for the transition from rapid temperature increase [Serbu and Maier, 1966] to the isothermal temperature distribution of interplanetary space far from the earth (see Figure 2). Because of these features of turbulent heat conduction, we adopt the turbulence process in the upper magnetosphere where the transition occurs from magnetosphere into interplanetary space.

Neglecting local and non-local heat sources at these altitudes, the energycontinuity equations for ions and electrons are

$$
\nabla_{11} j^{(i)}+\nabla_{\perp}{ }^{(i)}{ }^{(i)}=-L_{i e},
$$




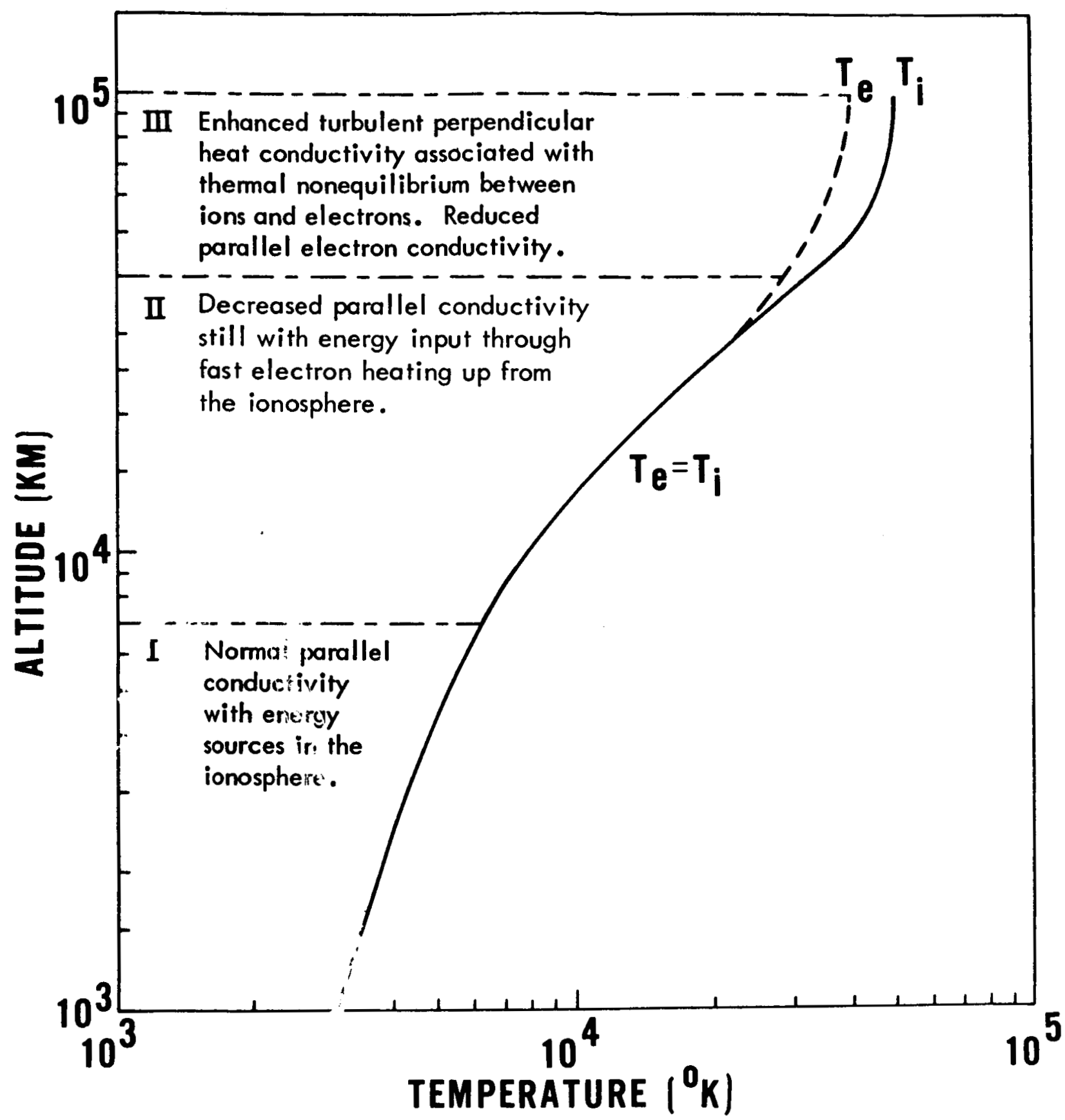

Figure 2. A model of the magnetospheric temperature structure. 


$$
\nabla_{11} \mathbf{j}^{(e)}+\nabla_{\perp} \mathbf{j}_{\perp}{ }^{(e)}=L_{i e}
$$

where the energy loss due to coulomb collisions between ions and electrons [Brace, Spencer, Dalgarno, 1965] is given as

$$
L_{i e}=\frac{K N_{e}^{2}\left(T_{i}-T_{e}\right)}{T_{e}^{3 / 2}}
$$

While the transverse turbulent heat conduction coefficients for electrons and ions are of the same order, the (reduced) parallel heat conduction coefficients differ by the factor $\left(m_{i} / m_{e}\right)^{1 / 2}$. The downward electron heat flux parallel to the magnetic field into the lower atmosphere is thus the dominant process of cooling the upper part of the magnetosphere. The ions will lose their energy (which is conducted from interplanetary space into the magnetosphere) mainly through coulomb collisions with electrons. Therefore, we expect the ion temperature to be larger than the electron temperature in this transition region.

In order to estimate this temperature difference and the associated energy input from interplanetary space, we consider the term lie in (31) as a heat sink which must compensate the perpendicular ion heat transfer. This leads to

$$
\frac{1}{r^{2}} \frac{\partial}{\partial r}\left[r^{2} j_{\perp}^{(i)}\right] \sim-K \frac{N_{e}^{2}\left(T_{i}-T_{e}\right)}{T_{e}^{3 / 2}}
$$

and after integration yields 


$$
\left[\frac{r_{1}^{2}}{r^{2}} j_{\perp}^{(i)}-j_{\perp 1}^{(i)}\right] \frac{3 r^{2}}{r^{3}-r_{1}^{3}} \sim \frac{K N_{e}{ }^{2}\left(T_{i}-T_{e}\right)}{T_{e}^{3 / 2}} .
$$

Equation 35 represents a relation between the ion-electron temperature difference, and the heat flux $j_{\perp}{ }^{(i)}$ from the interplanetary space into the magnetosphere. Assuming that at a height of about $r_{1}=7 r_{\theta}$ the heat flux is almost attenuated $\left(j_{\perp 1}^{(i)}=0\right)$, and adopting the values

$$
\begin{aligned}
& \mathrm{T}_{\mathrm{e}} \sim 4 \times 10^{4} \mathrm{~K}, \\
& \mathrm{~K} \sim 10^{-17}, \\
& \mathrm{~N} \sim 10 \mathrm{~cm}^{-3},
\end{aligned}
$$

we arrive with a reasonable temperature gradient of $0.1^{\circ} \mathrm{K} / \mathrm{km}$ and a corresponding energy flux

$$
j_{\perp}^{(i)}=-\kappa_{B} \frac{\partial T}{\partial r}=-10^{-10} \mathrm{erg} / \mathrm{cm}^{2} \sec
$$

at a temperature difference of

$$
\mathrm{T}_{1}-\mathrm{T}_{\mathrm{e}} \sim 10^{4}{ }^{\circ} \mathrm{K}
$$

This is consistent with Serbu and Maier's [1967] measurements.

Evidently such heat fluxes in the order of $10^{-10} \mathrm{erg} / \mathrm{cm}^{2} \mathrm{sec}$ are extremely small compared with those energy fluxes of $10^{-2}-10^{-3} \mathrm{erg} / \mathrm{cm}^{2} \mathrm{sec}$ that arise from the non-local magnetospheric heating due to the escape of fast electrons from the ionosphere. 
We conclude that the heat conduction between interplanetary space and magnetosphere, though sufficient to explain the observed thermal non-equilibrium in the upper magnetosphere makes a negligible contribution to the energy balance of the magnetosphere.

\section{CONC LUSIONS}

The foregoing results make it possible to construct a model of magnetospheric temperature distribution. Figure 2 shows a schematic equatorial temperature profile that extends from the bottom of the magnetosphere to interplanetary space.

Three regions are defined:

I. The lower magnetosphere, where the temperature distribution is determined by a normally high heat conduction due to high plasma densities $\left(10^{3}-10^{4} \mathrm{~cm}^{-3}\right)$, associated with heat received from the ionosphere. Here, the temperature profile is strongly affected by the observed latitudinal temperature increase up to 50 degrees geomagnetic latitude [Brace, Reddy, Mayr, 1966] which is related to ion compositional features [Mayr, Brace, Dunham, 1967] in conjunction with the energy balance of the lower F region.

II. The middle magnetosphere, where parallel heat conductivity can be largely decreased by low plasma densities $\left(10^{2}-10^{1} \mathrm{~cm}^{-3}\right)$, as previously discussed. The decrease in heat conduction and the consequent temperature increase along the field lines must be enough to counterbalance the temperature decrease with latitude observed at $1000 \mathrm{~km}$ above 50 degrees geomagnetic latitude [Brace, Reddy, Mayr, 1967] so that the temperature gradient increases with altitude in the equatorial plane. The energy input in this region may still be heating from the ionosphere. 
III. The upper magnetosphere, where perpendicular heat transfer becomes significant. Here, turbulence and plasma instabilities [Bohm, 1949, Tsuda, 1967] enormously increase the perpendicular heat transport coefficient. The plasma components therefore can carry some heat energy from the magnetopause and interplanetary space into the magnetosphere. Since the parallel heat conductivity is much greater for electrons than for ions, this incoming heat flux is transported mainly by electrons parallel to the magnetic field lines in the lower magnetosphere. The perpendicular heat conductivity of the ions is the same as that of the electrons; therefore, their heat energy is mainly transferred through coulomb collisions to the electrons. Thus ion temperature must be higher than electron temperature. With temperature gradients of $10^{-1}{ }^{\circ} \mathrm{K} / \mathrm{km}$, energy fluxes of $10^{-10} \mathrm{erg} / \mathrm{cm}^{2} \mathrm{sec}$ arise; these are large enough to explain the observed [Serbu and Maier, 1967] temperature differences of $\mathrm{T}_{\mathrm{i}}-\mathrm{T}_{\mathrm{e}} \sim 10^{4}{ }^{\circ} \mathrm{K}$. This supports the concept of turbulent heat transfer in the upper magnetosphere. But it also proves that thermal coupling between interplanetary space and magnetosphere is negligible compared with the energy input from escaping fast ionospheric electrons which produce heat fluxes of $10^{-2}-10^{-3} \mathrm{erg} / \mathrm{cm}^{2} \mathrm{sec}$.

We gratefully acknowledge valuable discussions with Dr. S. Bauer.

\section{REFERENCES}

Bohm, D., The characteristics of electric discharges in magnetic fields, edited by A. Guthrie and R. K. Wakerling, Chapter 2, Section 5, McGraw Hill, New York, 1949.

Brace, L. H. , Reddy, and H. Mayr, Global behavior of the ionosphere at $1000 \mathrm{~km}$ altitude, Geophys. Res. 72, 265-183, 1967. 
Brace, L. H., N. W. Spencer, and A. Dalgarno, Detailed Behavior of the Midlatitude Ionosphere from the Explorer XVII Satellite Planet Space Sci. 13, 647-666, 1965.

Serbu, G. P., and E. J. R. Maier, Thermal plasma measurements within the magnetosphere, Space Research VII, North Holland Publishing Company, Amsterdam, 1967.

Spitzer, L., Jr., to Physics of fully ionized gases, Interscience Publishers, Inc., New York, 1956.

Tamao, T., Temperature distribution in the magnetosphere, Report Ionosph. and Space Res. in Japan, 20, 1966, pp. 312-321.

Taylor, Jr., H.A., A. C. Brinton and L. R. Muenz, First results from OGO-C Ion Composition Experiment, Presented at the 47th Annual Meeting of AGU, March 1966.

Carpenter, D. L., and R. L. Smith, Whistler measurements of electron density in the magnetosphere, Rev. Geophys. 2, 415-441, 1964.

Geisler, J. E., and S. A. Bowhill, Exchange of energy between the ionosphere and the protonosphere, Atm. Terr. Phys. 27, 1119-1146, 1965.

Kaufman, A. N., Dissipative effects, in: Plasma physics in theory and application, edited by W. B. Kunkel, McGraw Hill, New York, 1966, p. 92 .

Mayr, M. G., L. H. Brace, and G. S. Dunham, On the ion composition and temperature in the topside ionosphere, J. Geophys. Res., 1967. 
Serbu, G. P., and E. J. R. Maier, Low energy electrons measured on IMP 2, Geophys. Res. 71, 3755-3766, 1966.

Taylor, Jr., H. A., H. C. Brinton, and C. R. Smith, Positive ion Composition in the magnetosphere, obtained from the OGO-A Satellite, J. Geophys. Res., 70, 5769-5771, 1965.

Tsuda, T., Effective viscosity of a streaming collision free plasma in a weakly turbulent magnetic field, AGU-Meeting, Washington, D. C., April 1967. 